\title{
De Novo Formation of Orbital Cavernous Malformation 9 Years After Surgical Management of Dural Arteriovenous Fistula in the Anterior Middle Fossa
}

\author{
-Case Report-
}

\author{
Shunsuke OMODAKA, Miki FujImURA, Toshiki EndO, \\ Takashi InOUE, Hiroaki SHIMIZU*, and Teiji TOMINAGA* \\ Department of Neurosurgery, Kohnan Hospital, Sendai, Miyagi; \\ * Department of Neurosurgery, Tohoku University Graduate School of Medicine, \\ Sendai, Miyagi
}

\begin{abstract}
A 30-year-old man presented with recurrent dural arteriovenous fistula (dAVF) associated with de novo orbital cavernous malformation (CM), manifesting as progressive left visual disturbance. He had undergone transarterial embolization and subsequent surgical management for a left anterior middle fossa dAVF 9 years previously. External carotid angiography showed recurrence of the dAVF. Magnetic resonance imaging revealed a well delineated intraorbital mass lesion with hypointense signal rim by $\mathrm{T}_{2}$-weighted imaging, adjacent to the shunting point of the recurrent dAVF. Transcranial subtotal removal of the intraorbital mass lesion through the orbitopterional approach revealed continuity between the mass lesion and the draining vein of the recurrent dAVF. The histological diagnosis was CM. His symptom was relieved postoperatively, and no regrowth was seen during the follow-up period of one year. The coexistence of recurrent dAVF with newly formed orbital CM is extremely rare, but may indicate the underlying mechanism of the formation of CMs and recurrent dAVF.
\end{abstract}

Key words: de novo cavernous malformation, dural arteriovenous fistula, orbital tumor

\section{Introduction}

Cerebral cavernous malformations (CMs) are generally considered to be biologically static, congenital lesions, ${ }^{1,13,18,23)}$ but recent reports have demonstrated the potential for more aggressive courses, such as rapid growth, repeated hemorrhage, recurrence after total removal, and de novo formation. ${ }^{6,16,20)}$ Therefore, cerebral CMs may include more dynamic and acquired lesions. Cerebral CMs are associated with other cerebrovascular lesions including vascular malformations, sinus thrombosis, ${ }^{5)}$ moyamoya disease, ${ }^{10)}$ and brain tumors. ${ }^{19)}$ However, association with dural arteriovenous fistula (dAVF) is extremely rare.$^{5,11)}$ Here we report a case of recurrent dAVF associated with orbital CM, which was not evident at the initial operation for dAVF.

\section{Case Report}

A 30-year-old man had suffered from progressive left visual disturbance for 2 years. He had undergone transarterial embolization and subsequent surgical treatment for middle temporal fossa dAVF 9 years previously (Fig. 1A).

Received June 18, 2009; Accepted August 17, 2009
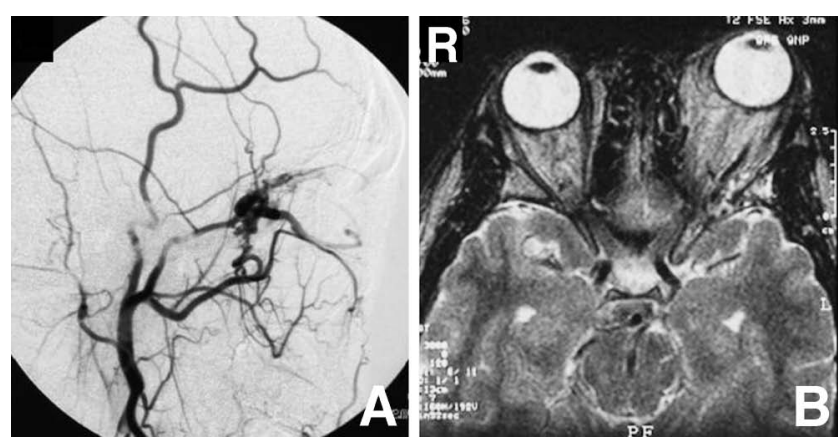

Fig. 1 Preoperative images obtained at the initial treatment 9 years previously. A: Left external carotid angiogram, lateral view, showing a left anterior middle fossa dural arteriovenous fistula mainly fed by the left middle meningeal artery and artery of the foramen rotundum, and draining into the superior orbital vein and inferior orbital vein. $B$ : Axial $T_{2}$-weighted magnetic resonance image demonstrating absence of orbital tumor.

$\mathrm{T}_{2}$-weighted imaging showed no intraorbital mass lesion (Fig. 1B).

On admission, clinical examination disclosed deteriora- 


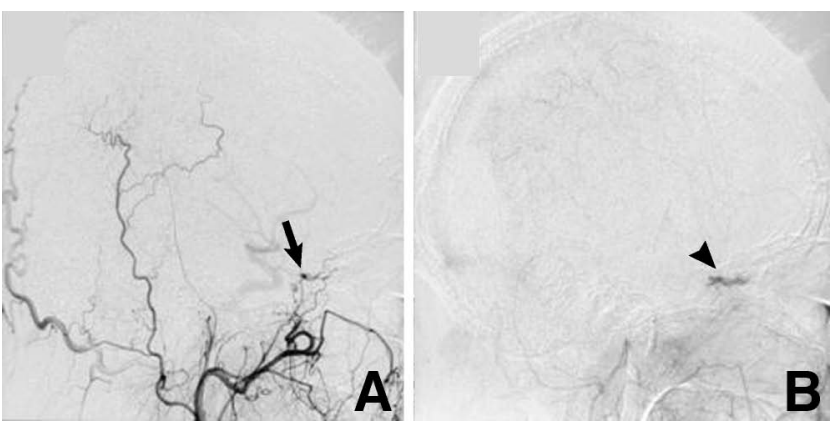

Fig. 2 Digital subtraction angiograms of the left external carotid artery on admission demonstrating the recurrent dural arteriovenous fistula in the left middle fossa. Arterial phase (A) showing early venous filling (arrow), and venous phase (B) showing pooling of the contrast material in the left superior orbital vein, forming the venous pouch (arrowhead).
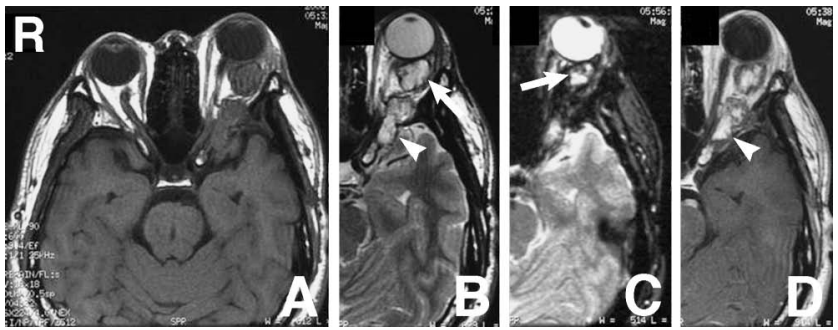

Fig. 3 Axial magnetic resonance images on admission demonstrating the well delineated multilobular mass lesion next to the venous pouch (arrowheads) in the left orbit. $T_{1}$-weighted image (A) showing the isointense mass extending from the frontal orbital region to the deep orbit near the optic canal. $T_{2}$-weighted image (B) showing the mass as a heterogeneous hyperintense lesion. $T_{2^{-}}(B)$ and $T_{2}{ }^{*}$-weighted (C) images demonstrating the hypointense rim (arrows) surrounding the mass. $T_{1}$-weighted image with contrast medium (D) showing the mass as partially enhanced.

tion of left visual acuity, non-pulsating and painless proptosis, and chemosis without cranial nerve paresis. Based on these findings, we suspected recurrent dAVF. Left external carotid angiography showed early venous filling from the artery of foramen rotundum, with pooling of the contrast material in the left superior orbital vein which forms the venous pouch (Fig. 2), suggesting the recurrence of dAVF. Magnetic resonance (MR) imaging demonstrated a well delineated mass lesion with multiple coalescent spherical components in the left orbit (Fig. 3), which was not evident at the initial diagnosis 9 years previously (Fig. 1B). The mass lesion extended from the frontal orbital region to the shunting point near the optic canal (Fig. 3D). $\mathrm{T}_{1}$ - and $\mathrm{T}_{2}$-weighted MR imaging showed the heterogeneous area as isointense and hyperintense, respectively (Fig. $3 \mathrm{~A}, \mathrm{~B}) . \mathrm{T}_{2^{-}}$and $\mathrm{T}_{2}{ }^{*}$-weighted $\mathrm{MR}$ imaging showed a hypointense rim surrounding the mass lesion (Fig. 3B, C). The lesion was enhanced partially with contrast material (Fig. 3D). No other mass lesion was demonstrated in the orbits or cranium. These findings indicated newly formed

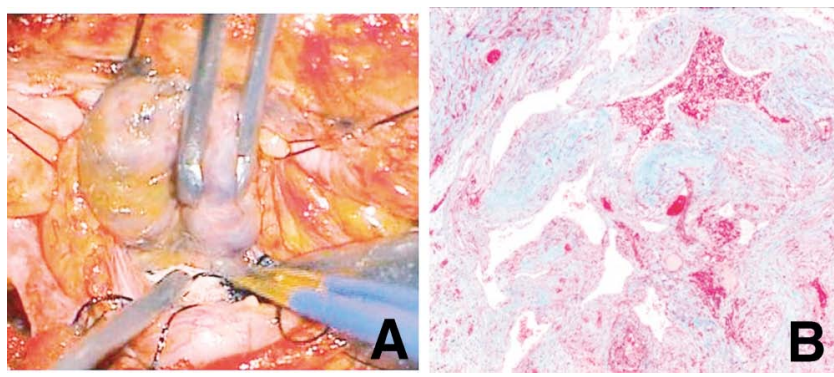

Fig. 4 A: Intraoperative photograph showing a dark reddish mulberry-like mass lesion embedded and encapsulated within the intraconal fatty tissue in the left orbit. B: Photomicrograph of the resected orbital cavernous malformation showing dilated vascular channels with thickened fibrotic walls and with no intervening brain parenchyma. Elastica-Masson stains, $\times 200$.
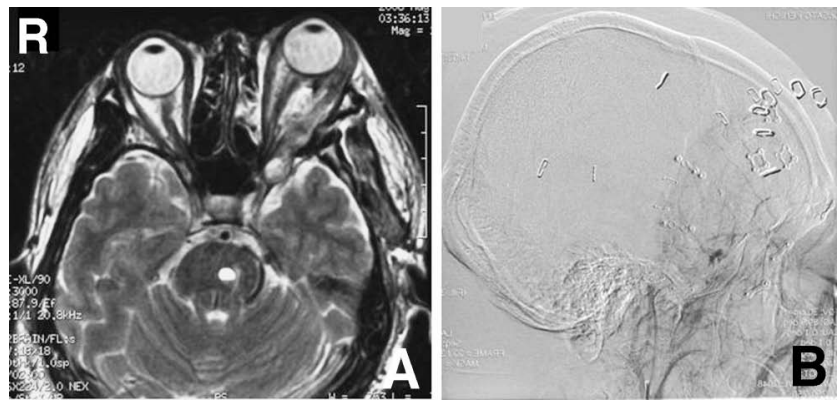

Fig. 5 A: Postoperative axial $T_{2}$-weighted magnetic resonance image showing subtotal removal of the mass. B: Postoperative left external carotid angiogram showing minimized venous pouch.

orbital CM. He denied any family history of seizures, stroke, or other symptoms of CMs.

Subtotal removal of the orbital mass lesion was performed through the left orbitopterional approach. Opening of the upper wall of the left orbit, and dissection of the superior and lateral rectus muscles revealed a dark reddish mulberry-like mass lesion embedded and encapsulated within the intraconal fatty tissue (Fig. 4A). Two obvious masses were removed, the deeper of which was continuous with the venous pouch in the deep orbit, and the vessels shunting to the venous pouch were coagulated. Histological examination showed clusters of sinusoidal vascular channels, with walls thickened by collagenous fiber, without intervening brain parenchyma (Fig. 4B). The histological features were consistent with a diagnosis of orbital CM.

The postoperative course was uneventful, and he was discharged without deterioration of his symptom 12 days after surgery. Postoperative MR imaging demonstrated that the tumor was subtotally removed (Fig. 5A) and angiography revealed a minimized venous pouch (Fig. 5B). Neuro-ophthalmologic examination 2 months after surgery showed improvement of the left visual impairment and regression of the proptosis and chemosis. No recur- 
rence was detected during the follow-up period of one year.

\section{Discussion}

The development of de novo cerebral CMs has an incidence of 0.2 to 0.4 lesions per patient per year. ${ }^{12,17)}$ Cerebral CMs can occur in sporadic or autosomaldominant inherited forms, and familial cases are known to be associated with multiple lesions, whereas non-familial cases usually consist of a single malformation. ${ }^{18)}$ The de novo formation of cerebral CMs is well recognized in patients with the familial form. Sporadic de novo cerebral CMs may occur after cranial radiation, ${ }^{14,15)}$ as a complication of preexisting venous malformations, ${ }^{15,20,21)}$ or as a novel, non-inherited mutation in the KRIT1 gene. ${ }^{9,16)}$ The present patient had no familial history of CMs or history of treatment with cranial radiation, and presented with recurrent $\mathrm{dAVF}$ and development of orbital CM. Initial surgery for the dAVF 9 years earlier included MR imaging that showed no indications of the orbital CM, thus suggesting de novo formation of orbital CM.

The association of dAVFs with de novo cerebral CMs is extremely rare, with only two previous cases. The first case of multiple de novo CMs occurred in a 3-month-old baby initially presenting with dAVF. ${ }^{11)}$ The second case of the development of multiple de novo vascular malformations including transverse sinus $\mathrm{dAVF}$ and posterior fossa CMs followed surgery for acoustic neuroma. ${ }^{5}$ Hemodynamic change in the capillary-venous side of the vascular network may contribute to the de novo formation of CMs. More than two thirds of cases of periorbital lymphatic malformations are associated with intracranial vascular anomalies. ${ }^{3)}$ In the present case, the recurrence of anterior middle fossa dAVF and orbital CMs were simultaneously detected 9 years after the initial treatment, when no intraorbital lesion was detected by MR imaging, suggesting de novo formation of CMs with dAVF recurrence. Whether this association represents a true cause-andeffect relationship or is simply a coincidental expression of an inappropriately formed cerebral vasculature remains unclear.

The pathogenesis of cerebral CMs is still unknown. Recent immunohistochemical studies have demonstrated the expression of biological markers in cerebral CMs, such as proliferating cell nuclear antigen, MIB-1, Flk-1, vascular endothelial growth factor, hypoxia-inducible factor- $1 \alpha$, and matrix metalloproteinase-9, indicating cerebral CMs have proliferating and neoangiogenetic characters. ${ }^{8,7,20)}$ Mixed cerebrovascular malformations with pathological features of more than one type of malformation within the same lesion, most frequently found with venous malformations, are now being recognized with increasing frequency. ${ }^{22)}$ The physical proximity of the different subtypes of vascular malformations and the pathological heterogeneity within each lesion may support the idea of a common origin of distinct vascular malformations., ${ }^{2,14,21)}$ Elevated venous pressure within the territory of a venous malformation may lead to the biological cascade of cerebral CM development.7)
Cerebral CMs associated with venous malformations are more likely to be acquired rather than true congenital vascular malformations. ${ }^{4)}$ The mechanism of formation of de novo cerebral CMs associated with other vascular malformation may involve microhemorrhage caused by abnormal vascular beds, which may induce hemodynamic disturbance or may be fragile, resulting in reactive angiogenesis with new vessel formation and coalescence. ${ }^{2)}$ Therefore, the development of de novo CMs is apparently promoted by several angiogenic factors stimulated by diapedetic microhemorrhages resulting from the fluctuating venous pressure, so-called "hemorrhagic angiogenetic proliferation." ${ }^{2,21)}$ Alternatively, hemodynamic disturbance, such as venous outflow restriction, might open preexisting arteriovenous connections, resulting in minute arteriovenous shunts that can enlarge over time. ${ }^{2,15)}$ In the present case, the recurrent dAVF with venous pouch might indicate venous hypertension and represent the main mechanism responsible for fluctuating venous pressure, microhemorrhage, endothelial growth factor activation, and subsequent formation of CMs. The fluctuating venous pressure might also be responsible for the recurrence and/or growth of the dAVF.

Coexistence of recurrent dAVF with de novo orbital CM is extremely rare, but may indicate the underlying mechanism of formation of CMs and recurrent dAVF.

\section{References}

1) Aiba T, Tanaka R, Koike T, Kameyama S, Takeda N, Komata $\mathrm{T}$ : Natural history of intracranial cavernous malformations. J Neurosurg 83: 56-59, 1995

2) Awad IA, Robinson JR Jr, Mohanty S, Estes ML: Mixed vascular malformations of the brain: Clinical and pathogenetic considerations. Neurosurgery 33: 179-188, 1993

3) Bisdorff A, Mulliken JB, Carrico J, Robertson RL, Burrows $\mathrm{PE}$ : Intracranial vascular anomalies in patients with periorbital lymphatic and lymphaticovenous malformations. AJNR Am J Neuroradiol 28: 335-341, 2007

4) Campeau NG, Lane JI: De novo development of a lesion with the appearance of a cavernous malformation adjacent to an existing developmental venous anomaly: case report. AJNR Am J Neuroradiol 26: 156-159, 2005

5) Desal HA, Lee SK, Kim BS, Raoul S, Tymianski M, TerBrugge KG: Multiple de novo vascular malformations in relation to diffuse venous occlusive disease: a case report. Neuroradiology 47: 38-42, 2005

6) Detwiler PW, Porter RW, Zabramski JM, Spetzler RF: De novo formation of a central nervous system cavernous malformation: implications for predicting risk of hemorrhage. Case report and review of the literature. J Neurosurg 87: 629-632, 1997

7) Dillon WP: Cryptic vascular malformations: controversies in terminology, diagnosis, pathophysiology, and treatment. AJNR Am J Neuroradiol 18: 1839-1846, 1997

8) Fujimura $M$, Watanabe $M$, Shimizu H, Tominaga T: Expression of matrix metalloproteinases (MMPs) and tissue inhibitor of metalloproteinase (TIMP) in cerebral cavernous malformations: immunohistochemical analysis of MMP-2, -9 and TIMP-2. Acta Neurochir (Wien) 149: 179-183, 2007

9) Gault J, Sarin H, Awadallah NA, Shenkar R, Awad IA: Pathobiology of human cerebrovascular malformations: basic mechanisms and clinical relevance. Neurosurgery 55: 
$1-16,2004$

10) Januschek E, Fujimura $M$, Mugikura $S$, Tominaga $T$ : Progressive moyamoya syndrome associated with de novo formation of the ipsilateral venous and contralateral cavernous malformations: case report. Surg Neurol 69: 423-427, 2008

11) Janz C, Meisel J, Brock M, Lasjaunias P: De novo appearance of three cavernomas in an infant with dural AV shunts. Interventional Neuroradiology 4: 85-90, 1998

12) Jung KH, Chu K, Jeong SW, Park HK, Bae HJ, Yoon BW: Cerebral cavernous malformations with dynamic and progressive course: correlation study with vascular endothelial growth factor. Arch Neurol 60: 1613-1618, 2003

13) Kondziolka D, Lunsford LD, Kestle JRW: The natural history of cerebral cavernous malformations. J Neurosurg 83: 820-824, 1995

14) Pozzati E, Acciarri N, Tognetti F, Marliani F, Giangaspero F: Growth, subsequent bleeding, and de novo appearance of cerebral cavernous angiomas. Neurosurgery 38: 662-669, 1996

15) Pozzati E, Giangaspero F, Marliani F, Acciarri N: Occult cerebrovascular malformations after irradiation. Neurosurgery 39: 677-684, 1996

16) Pozzati E, Marliani AF, Zucchelli M, Foschini MP, Dall'Olio M, Lanzino G: The neurovascular triad: mixed cavernous, capillary, and venous malformations of the brainstem. J Neurosurg 107: 1113-1119, 2007

17) Reid PJ, Campbell SS, Vates GE, Allende R: Extreme de novo appearance of cerebral cavernous malformations: case report. Neurosurgery 62: 969-970, 2008

18) Rigamonti D, Hadley MN, Drayer BP, Johnson PC, HoenigRigamonti K, Knight JT, Spetzler RF: Cerebral cavernous malformations: Incidence and familial occurrence. $\mathrm{N}$ Engl J Med 319: 343-347, 1988

19) Sakata H, Fujimura $M$, Watanabe $M$, Tominaga T: Association of cavernous malformation within vestibular schwannoma: immunohistochemical analysis of matrix metalloproteinase-2 and -9. Neurol Med Chir (Tokyo) 47: 509-512, 2007

20) Sure U, Freman S, Bozinov O, Benes L, Siegel AM, Bertalanffy $\mathrm{H}$ : Biological activity of adult cavernous malformations: a study of 56 patients. J Neurosurg 102: 342-347, 2005

21) Wilson CB: Cryptic vascular malformations. Clin Neurosurg 38: 49-84, 1992

22) Wurm G, Schnizer M, Fellner FA: Cerebral cavernous malformations associated with venous anomalies: surgical considerations. Neurosurgery 57: 42-58, 2005

23) Zabramski JM, Wascher TM, Spetzler RF, Johnson B, Golfinos J, Drayer BP, Brown B, Rigamonti D, Brown G: The natural history of familial cavernous malformations: Results of an ongoing study. J Neurosurg 80: 422-432, 1994

Address reprint requests to: Miki Fujimura, M.D., Department of Neurosurgery, Kohnan Hospital, 4-20-1 Nagamachi-Minami, Taihaku-ku, Sendai 982-0012, Japan.

e-mail: fujimur@kohnan-sendai.or.jp 